\title{
Palmoplantar Eruption in a Patient With Mercury Poisoning
}

\author{
Michelle A. McNally, BSN; Helena Jenkinson, MD; Saisindhu Narala, MD; Megan Rogge, MD
}

\section{PRACTICE POINTS}

- The dermatologic and histologic presentation of mercury exposure may be nonspecific, requiring a high degree of clinical suspicion to make a diagnosis.

- Mercury exposure should be included in the differential diagnosis in patients presenting with a rash of the palms and soles, especially in young patients with systemic symptoms.

Mercury poisoning is a rare event that can present with a variety of nonspecific systemic symptoms, making it difficult to diagnose. Dermatologic manifestations of mercury exposure may be variable and include pink disease (acrodynia), mercury exanthem, contact dermatitis, and cutaneous granulomas. We present the case of an 18-year-old woman with a palmoplantar eruption associated with tachycardia, hyperhidrosis, myalgia, paresthesia, and muscle fasciculations. Physical examination demonstrated poorly demarcated pink macules coalescing into patches on the left palm, right wrist, and soles. A punch biopsy was nonspecific, showing acanthosis and orthokeratosis with mild inflammation. Elevated urine and serum mercury levels confirmed a diagnosis of mercury poisoning. This case highlights the importance of consideration of mercury poisoning in the differential diagnosis for acral eruptions, especially in the presence of systemic symptoms and known risk factors.

Cutis. 2020;106:265-267.

M ercury poisoning affects multiple body systems, leading to variable clinical presentations. Mercury intoxication at low levels frequently presents with weakness, fatigue, weight loss, and abdominal pain. At higher levels of mercury intoxication, tremors and neurologic dysfunction are more prevalent. ${ }^{1}$ Dermatologic manifestations of mercury exposure vary and include pink disease (acrodynia), mercury exanthem, contact dermatitis, and cutaneous granulomas. Untreated mercury poisoning may result in severe complications, including renal tubular necrosis, pneumonitis, persistent neurologic dysfunction, and fatality in some cases. ${ }^{1,2}$

Pink disease is a rare disease that typically arises in infants and young children from chronic mercury exposure. ${ }^{3}$ We report a unique presentation of pink disease occurring in an 18-year-old woman following mercury exposure.

\section{Case Report}

An 18-year-old woman who was previously healthy presented to the hospital for evaluation of body aches and back pain. She reported a transient rash on the torso 2 weeks prior, but at the current presentation, only the distal upper and lower extremities were involved. A review of systems revealed myalgia, most severe in the lower back; muscle spasms; stiffness in the fingers; abdominal pain; constipation; paresthesia in the hands and feet; hyperhidrosis; and generalized weakness.

Vitals on admission revealed tachycardia (112 beats per minute). Physical examination revealed the patient was pale and fatigued; she appeared to be in pain, with observable facial grimacing and muscle spasms in the legs. She had poorly demarcated pink macules and papules scattered on the left palm (Figure 1), right forearm, right wrist, and dorsal aspects of the feet including the soles. A few pinpoint pustules were present on the left fifth digit.

From the Department of Dermatology, John P. and Kathrine G. McGovern Medical School at the University of Texas Health Science Center at Houston. The authors report no conflict of interest.

Correspondence: Michelle A. McNally, BSN, 6431 Fannin St, Houston, TX 77030 (michelle.a.mcnally@uth.tmc.edu).

doi:10.12788/cutis.0113 


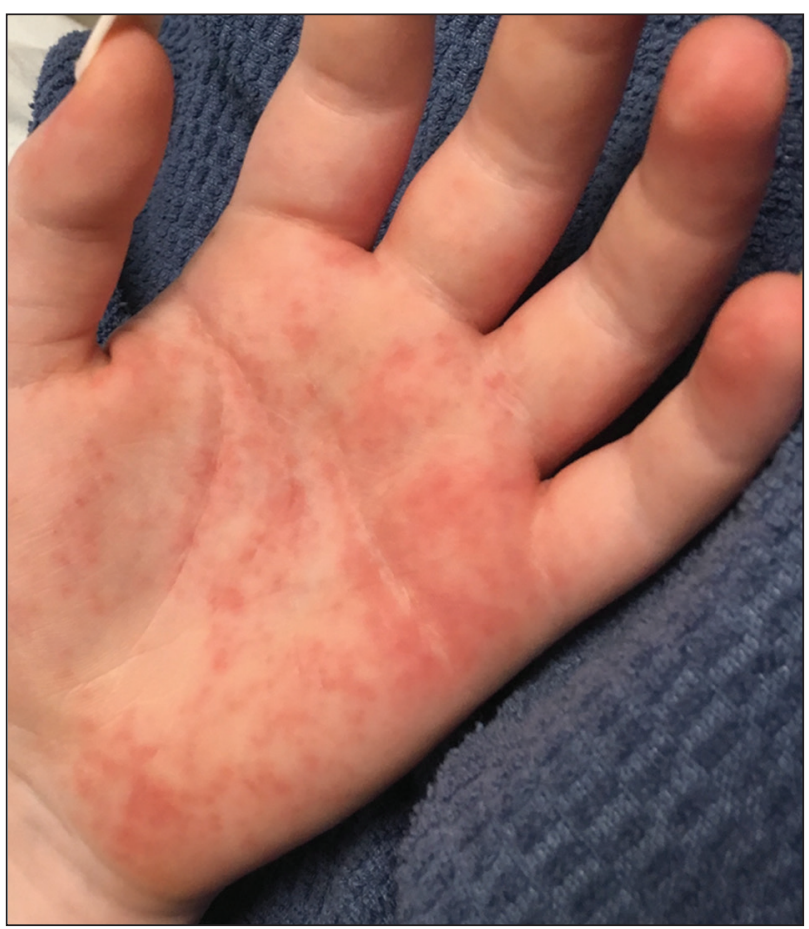

FIGURE 1. Left palm with erythematous blanching macules coalescing into patches.

An extensive workup was initiated to rule out infectious, autoimmune, or toxic etiologies. Two 4- $\mathrm{mm}$ punch biopsies of the left palm were performed for hematoxylin and eosin staining and tissue culture. Findings on hematoxylin and eosin stain were nonspecific, showing acanthosis, orthokeratosis, and a mild interface and perivascular lymphocytic infiltrate (Figure 2); superficial bacterial colonization was present, but the tissue culture was negative.

Laboratory studies showed mild transaminitis, and stool was positive for Campylobacter antigen. Electromyography showed myokymia (fascicular muscle contractions). A heavy metal serum panel and urine screen were positive for elevated mercury levels, with a serum mercury level of $23 \mu \mathrm{g} / \mathrm{L}$ (reference range, $0.0-14.9 \mu \mathrm{g} / \mathrm{L}$ ) and a urine mercury level of $76 \mu \mathrm{g} / \mathrm{L}$ (reference range, $0-19 \mu \mathrm{g} / \mathrm{L}$ ).

Upon further questioning, it was discovered that the patient's brother and neighbor found a glass bottle containing mercury in their house 10 days prior. They played with the mercury beads with their hands, throwing them around the room and spilling them around the house, which led to mercury exposure in multiple individuals, including our patient. Of note, her brother and neighbor also were hospitalized at the same time as our patient with similar symptoms.

A diagnosis of mercury poisoning was made along with a component of postinfectious reactive arthropathy due to Campylobacter. The myokymia and skin eruption
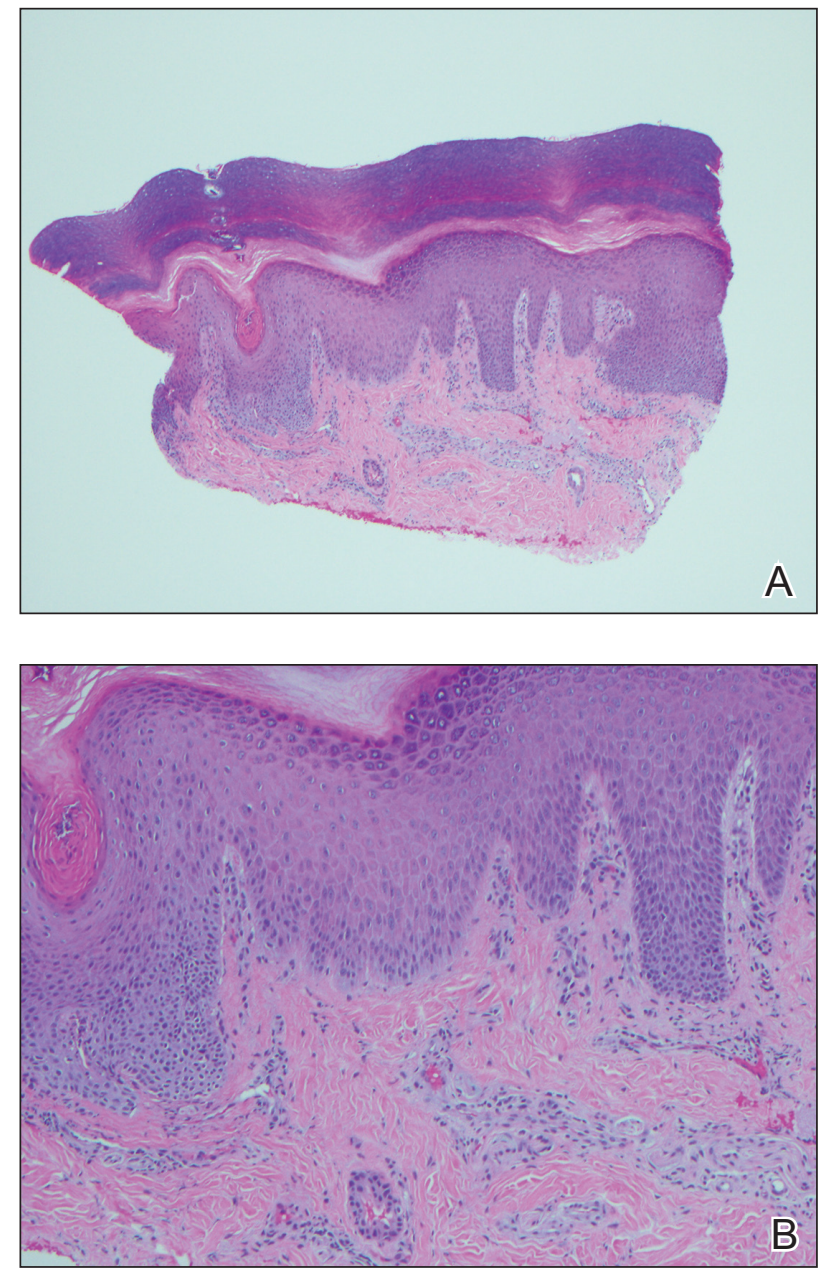

FIGURE 2. A, A punch biopsy from acral skin demonstrated irregular acanthosis, orthokeratosis, and a mild perivascular lymphocytic infiltrate $(\mathrm{H} \& \mathrm{E}$, original magnification $\times 20)$. B, Higher magnification showed few neutrophils present within a loosely lichenoid infiltrate, resembling lichenoid dermatitis (H\&E, original magnification $\times 100$ ).

were believed to be secondary to mercury poisoning. The patient was started on ciprofloxacin (750 mg twice daily), intravenous immunoglobulin for Campylobacter, a 2-week treatment regimen with the chelating agent succimer (500 mg twice daily) for mercury poisoning, and a 3-day regimen of pulse intravenous steroids (intravenous methylprednisolone $500 \mathrm{mg}$ once daily) to reduce inflammation. Repeat mercury levels showed a downward trend, and the rash improved with time. All family members were advised to undergo testing for mercury exposure.

\section{Comment}

Manifestations of Mercury Poisoning-Dermatologic manifestations of mercury exposure are varied. The most common-allergic contact dermatitis-presents after repeat systemic or topical exposure. ${ }^{4}$ Mercury exanthem is an acute systemic contact dermatitis most commonly triggered by mercury vapor inhalation. It manifests as an 
erythematous maculopapular eruption predominantly involving the flexural areas and the anterior thighs in a V-shaped distribution. ${ }^{5}$ Purpura may be seen in severe cases. Cutaneous granulomas after direct injection of mercury also have been reported as well as cutaneous hyperpigmentation after chronic mercury absorption. ${ }^{6}$

Presentation of Pink Disease-Pink disease occurs in children after chronic mercury exposure. It was a common pediatric disorder in the 19th century due to the presence of mercury in certain anthelmintics and teething powders. ${ }^{7}$ However, prevalence drastically decreased after the removal of mercury from these products. ${ }^{3}$ Although pink disease classically was associated with mercury ingestion, cases also occurred secondary to external application of mercury. ${ }^{7}$ Additionally, in 1988 a case was reported in a 14-month-old girl after inhalation of mercury vapor from a spilled bottle of mercury. ${ }^{3}$

Pink disease begins with pink discoloration of the fingertips, nose, and toes, and later progresses to involvement of the hands and feet. Erythema, edema, and desquamation of the hands and feet are seen, along with irritability and autonomic dysfunction that manifests as profuse perspiration, tachycardia, and hypertension. ${ }^{3}$

Diagnosis of Pink Disease-The differential diagnosis of palmoplantar rash is broad and includes rickettsial disease; syphilis; scabies; toxic shock syndrome; infective endocarditis; meningococcal infection; hand-footand-mouth disease; dermatophytosis; and palmoplantar keratodermas. The involvement of the hands and feet in our patient, along with hyperhidrosis, tachycardia, and paresthesia, led us to believe that her condition was a variation of pink disease. The patient's age at presentation (18 years) was unique, as it is atypical for pink disease. Although the polyarthropathy was attributed to Campylobacter, it is important to note that high levels of mercury exposure also have been associated with polyarthritis, ${ }^{8}$ polyneuropathy, ${ }^{4}$ and neuromuscular abnormalities on electromyography. ${ }^{4}$ Therefore, it is possible that the presence of these symptoms in our patient was either secondary to or compounded by mercury exposure.

Mercury Poisoning-Diagnosis of mercury poisoning can be made by assessing blood, urine, hair, or nail concentrations. However, as mercury deposits in multiple organs, individual concentrations do not correlate with total-body mercury levels. ${ }^{1}$ Currently, no universal diagnostic criteria for mercury toxicity exist, though a provocation test with the chelating agent 2,3-dimercaptopropanesulfonate is considered reliable in assessing total-body mercury burden. ${ }^{1}$

Elemental mercury, as found in some thermometers, dental amalgams, and electrical appliances (eg, certain switches, fluorescent light bulbs), can be converted to inorganic mercury in the body. ${ }^{9}$ Elemental mercury is vaporized at room temperature; the predominant route of exposure is by subsequent inhalation and lung absorbtion. ${ }^{10}$ Cutaneous absorption of high concentrations of elementary mercury in either liquid or vapor form may occur, though the rate is slow and absorption is poor. In cases of accidental exposure, contaminated clothing should be removed and immediately decontaminated or disposed. Exposed skin should be washed with a mild soap and water and rinsed thoroughly. ${ }^{10}$

The treatment of inorganic mercury poisoning is accomplished with the chelating agents succimer, dimercaptopropanesulfonate, dimercaprol, or D-penicillamine. ${ }^{1}$ In symptomatic cases with high clinical suspicion, the first dose of chelation treatment should be initiated early without delay for laboratory confirmation, as treatment efficacy decreases with an increased interim between exposure and onset of chelation. ${ }^{11}$ Combination chelation therapy also may be used in treatment. Plasma exchange or hemodialysis are treatment options for extreme, lifethreatening cases. ${ }^{1}$

\section{Conclusion}

Mercury exposure should be included in the differential diagnosis of patients presenting with a rash on the palms and soles, especially in young patients with systemic symptoms. A high level of suspicion and a thorough history can prevent a delay in treatment and an unnecessarily extensive and expensive workup. An emphasis on early diagnosis and treatment is important for optimal outcomes and can prevent the severe and potentially devastating consequences of mercury toxicity.

\section{REFERENCES}

1. Bernhoft RA. Mercury toxicity and treatment: a review of the literature. J Environ Public Health. 2012;2012:460508.

2. Kamensky OL, Horton D, Kingsley DP, et al. A case of accidental mercury intoxication. J Emerg Med. 2019;56:275-278.

3. Dinehart SM, Dillard R, Raimer SS, et al. Cutaneous manifestations of acrodynia (pink disease). Arch Dermatol. 1988;124:107-109.

4. Malek A, Aouad K, El Khoury R, et al. Chronic mercury intoxication masquerading as systemic disease: a case report and review of the literature. Eur J Case Rep Intern Med. 2017;4:000632.

5. Nakayama H, Niki F, Shono M, et al. Mercury exanthem. Contact Dermatitis. 1983;9:411-417.

6. Boyd AS, Seger D, Vannucci S, et al. Mercury exposure and cutaneous disease. J Am Acad Dermatol. 2000;43:81-90.

7. Warkany J. Acrodynia-postmortem of a disease. Am J Dis Child. 1966;112:147-156.

8. Karataş GK, Tosun AK, Karacehennem E, et al. Mercury poisoning: an unusual cause of polyarthritis. Clin Rheumatol. 2002;21:73-75.

9. Mercury Factsheet. Centers for Disease Control and Prevention website. https://www.cdc.gov/biomonitoring/Mercury_FactSheet.html. Reviewed April 7, 2017. Accessed October 21, 2020.

10. Medical management guidelines for mercury. Agency for Toxic Substances \& Disease Registry website. https://www.atsdr.cdc .gov/MMG/MMG.asp?id=106\&tid=24. Update October 21, 2014. Accessed September 11, 2020.

11. Kosnett MJ. The role of chelation in the treatment of arsenic and mercury poisoning. J Med Toxicol. 2013;9:347-354. 